\title{
TRATAMENTO DE HEMORRAGIA INTESTINAL GRAVE DECORRENTE DE HIPERTENSÃO PORTA, POR MEIO DE ESPLENECTOMIA SUBTOTAL E ANASTOMOSE ESPLENORRENAL PROXIMAL
}

\author{
TREATMENT OF SEVERE INTESTINAL BLEEDING DUE TO PORTAL HYPERTENSION \\ BY MEAN OF SUBTOTAL SPLENECTOMY AND CENTRAL SPLENORENAL SHUNT
}

\author{
Andy Petroianu - TCBC-MG'; João Baptista Rezende Neto - TCBC-MG²
}

\begin{abstract}
RESUMO: Objetivo: Um dos maiores desafios no tratamento da hipertensão porta é o sangramento de varizes intestinais. O objetivo deste artigo é apresentar um novo tratamento para hemorragia entérica grave provocada por hipertensão porta cirrótica, utilizando a esplenectomia subtotal combinada com derivação esplenorrenal proximal. Técnica: Uma paciente de 64 anos apresentou sangramento intenso proveniente de varizes em alça jejunal em Y de Roux, para derivação biliodigestiva, após lesão coledociana, complicada com cirrose hepática. O procedimento consistiu em esplenectomia subtotal, preservando o pólo superior do baço, suprido apenas pelos vasos esplenogástricos, e anastomose término-lateral da veia esplênica, em sua extremidade proximal, à veia renal esquerda. Resultados: Esse procedimento foi seguro, sem sangramento maior, dificuldade técnica ou complicação. No acompanhamento de 30 meses, a paciente não teve novos episódios de sangramento, a cirrose não avançou e, atualmente, encontrase bem, sob controle. Conclusão: A esplenectomia subtotal combinada com derivação esplenorrenal proximal é uma boa alternativa operatória para tratar hemorragia proveniente de varizes intestinais, decorrentes de hipertensão porta (Rev. Col. Bras. Cir. 2008; 35(4): 264-268)
\end{abstract}

Descritores: Esplenectomia; Derivação Esplenorrenal cirúrgica; Hemorragia gastrointestinal; Varizes esofágicas e gástricas; Hipertensão portal.

\section{INTRODUÇÃO}

Em decorrência de obstrução ao fluxo sangüíneo do sistema porta, ocorre estase, com conseqüente elevação da pressão do sistema venoso, em um quadro conhecido como hipertensão porta, Por ser um fluxo avalvulado, essa elevação pressórica ocorre em todas as veias que direta ou indiretamente drenam para a veia porta. Como as tributárias menores desse sistema não estão preparadas para suportar essa hipertensão, elas dilatam-se e tornam-se tortuosas, formando varizes em todo o sistema digestório, dede o esôfago até o plexo hemorroidário interno.

Essa situação é bem suportada pelo organismo e, na maioria dos casos não se acompanha de repercussão clínica alguma. No entanto, em decorrência das doenças que geram esse quadro hipertensivo, podem ocorrer distúrbios mais graves, incluindo hemorragia digestiva. Por características venosas peculiares, a quase totalidade desses sangramentos ocorre no esôfago inferior, cárdia e fundo gástrico.

A causa mais comum de hipertensão porta é a cirrose hepática. Aproximadamente metade dos pacientes cirróticos portadores de hipertensão porta desenvolve varizes no esôfago e no estômago. Segundo dados da literatura, cerca de um terço deles poderão evoluir com pelo menos um episó- dio de sangramento por varizes ${ }^{1,2,3}$. Por outro lado, a incidência de sangramentos provenientes de varizes localizadas fora dessa região é muito baixo, girando em torno de $1 \%$ e $5 \%$. Os locais mais frequientes de hemorragia não-esofagogástrica são os estomas ${ }^{6-10}$. Sangramentos em outras regiões relacionadas com a hipertensão porta são excepcionais.

Outra complicação da estase sangüínea no sistema porta é a esplenomegalia, conhecida sob o nome impróprio de hiperesplenismo, em decorrência os valores hematológicos baixos, apesar de não serem acompanhados por repercussões clínicas e não necessitar de remoção do baço para a resolução do problema ${ }^{11}$. O represamento de sangue no baço provoca o seu crescimento e aumento da função de armazenamento dos elementos sangüíneos, porém sem destruí-los e os disponibilizando em caso de necessidade, portanto sem distúrbios na coagulação o defesa orgânicas.

O desconforto provocado pela esplenomegalia acompanhada de pancitopenia faz com que muitos médicos indiquem a retirada completa do baço como parte do tratamento da hipertensão porta. Esse procedimento reduz apenas temporariamente a pressão porta e pode prevenir o sangramento, porém priva o doente das importantes funções esplênicas, com conseqüentes riscos de sepse, hipercoagulabilidade sangüínea, febre, sobrecarga funcional hepática, etc.

1. Professor Titular do Departamento de Cirurgia da Faculdade de Medicina da UFMG; Docente Livre em Técnica Operatória e Cirurgia Experimental da Escola Paulista de Medicina, UNIFESP; Docente Livre em Gastroenterologia Cirúrgica da Faculdade de Medicina de Ribeirão Preto, USP;

Doutor em Fisiologia e Farmacologia - Instituto de Ciências Biológicas, UFMG, Pesquisador IA do CNPq.

2. Professor Adjunto do Departamento de Cirurgia da Faculdade de Medicina da UFMG, Doutor em Cirurgia.

Recebido em 11/03/2008

Aceito para publicação em 14/04/2008

Conflito de interesses: nenhum

Fonte de financiamento: nenhuma

Trabalho realizado na Santa Casa de Misericórdia de Belo Horizonte - MG. 
Em 1979, teve início uma linha de estudos sobre esplenectomia subtotal, com preservação do pólo superior do baço, visando ao tratamento de diversas afecções esplênicas. Desde 1984, tem sido realizada com sucesso essa operação isolada ou associada a outros procedimentos, como, nos casos de hipertensão porta, complementada por desconexão portavarizes ou derivação esplenorrenal centralizada ${ }^{11-20}$. Essa operação já foi realizada em mais de 230 pacientes, sem complicações atribuídas ao remanescente esplênico, mesmo após longos períodos de acompanhamento ${ }^{21-25}$.

Este artigo descreve a aplicação e a técnica da esplenectomia subtotal associada a derivação esplenorrenal proximal em uma paciente portadora de cirrose biliar e hipertensão porta, que apresentou hemorragia vultosa proveniente de varizes localizadas em alça jejunal de Y de Roux.

\section{NOTA TÉCNICA}

Uma paciente de 49 foi operada para tratamento de colecistite aguda. Devido a lesão do colédoco, durante o ato operatório, realizou-se coledocojejunostomia em Y de Roux. Em decorrência de vários episódios de colangite pós-operatória, a doença evoluiu para cirrose hepática.

Após 15 anos do procedimento inicial, a paciente apresentou hematoquesia. A endoscopia digestiva alta e a colonoscopia não mostraram varizes ou outras afecções que pudessem ser responsabilizadas por essa manifestação. Após duas semanas, o sangramento tornou-se muito intenso, acompanhado de choque e queda da hemoglobina para 5,8 g/dl. A arteriografia mesentérica mostrou sangramento na topografia da flexura colônica direita e trombose do ramo direito da veia porta, com hiperfluxo na veia mesentérica superior (Figura 1).

A paciente foi submetida a laparotomia exploradora, na qual se encontrou cirrose hepática, confirmada por biopsia, esplenomegalia e a presença de sangue no lúmen da alça do Y. Por meio de enteroscopia peroperatória, evidenciou-se sangramento proveniente de varizes intraluminares dessa alça. Através de enterotomia, as varizes foram suturadas.

Ao final da primeira semana pós-operatória, a paciente apresentou novo sangramento digestivo intenso e foi encaminhada para o nosso Serviço. Para tratar esse sangramento, decorrente da hipertensão porta, decidiu-se reduzir a pressão sangüínea no sistema venoso mesentérico, incluindo nas varizes da alça jejunal, por meio de derivação esplenorrenal proximal. Para evitar a perda das funções esplênicas, em vez de remover-se todo o baço, optou-se pela esplenectomia subtotal.

No espaço retrogástrico, a artéria esplênica foi ligada. Em seguida, o baço foi completamente mobilizado para o campo operatório. Os vasos para o pólo inferior do órgão foram ligados e seccionados. A artéria esplênica e os vasos do pedículo para o pólo superior do baço foram também ligados e seccionados. A veia esplênica foi cuidadosamente dissecada e ligada junto ao hilo esplênico.

O baço foi seccionado em cunha, preservando seu pólo superior suprido apenas pelos vasos esplenogástricos. Após realizar-se a hemostasia dos vasos do parênquima esplênico, por meio de sutura com fios de categute 3-0 e 4-0, a cápsula do baço foi suturada em chuleio, utilizando fio de categute cromado 3-0.O remanescente esplênico foi reposicionado em seu leito e fixado com um fio de categute cromado 3-0. (Figura 2A)

Em seguida, a veia esplênica foi dissecada até próximo da confluência com a veia mesentérica superior, onde também penetrava a veia mesentérica inferior, como tributária da veia esplênica, tendo sido ambas as mesentéricas preservadas. A veia renal esquerda foi identificada e dissecada na região onde a veia supra-renal penetra nela. Essa tributária foi ligada e seccionada. A veia renal foi aberta em torno da entrada da veia supra-renal, após a colocação de uma pinça de Satinsky. Confeccionou-se a anastomose término-lateral entre a veia esplênica e a veia renal esquerda, com fio de polipropileno 6-0. (Figura 2B)
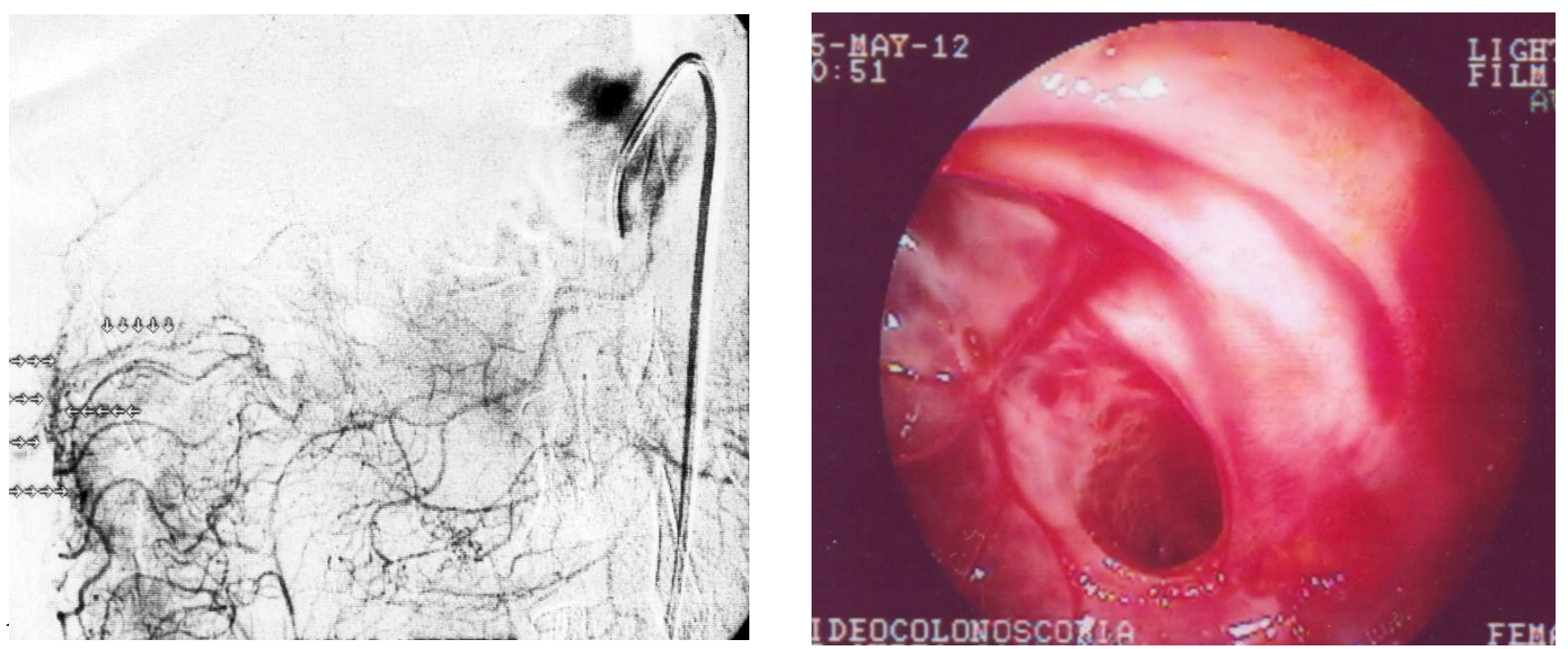

Figura 1 - Exame pré-operatório mostrando o sangramento na alça jejunal em Y de Roux. A - Arteriografia, que mostra contraste extravasando ao nível da flexura colônica direita. B - Vista endoscópica do sangramento em alça jejunal. 

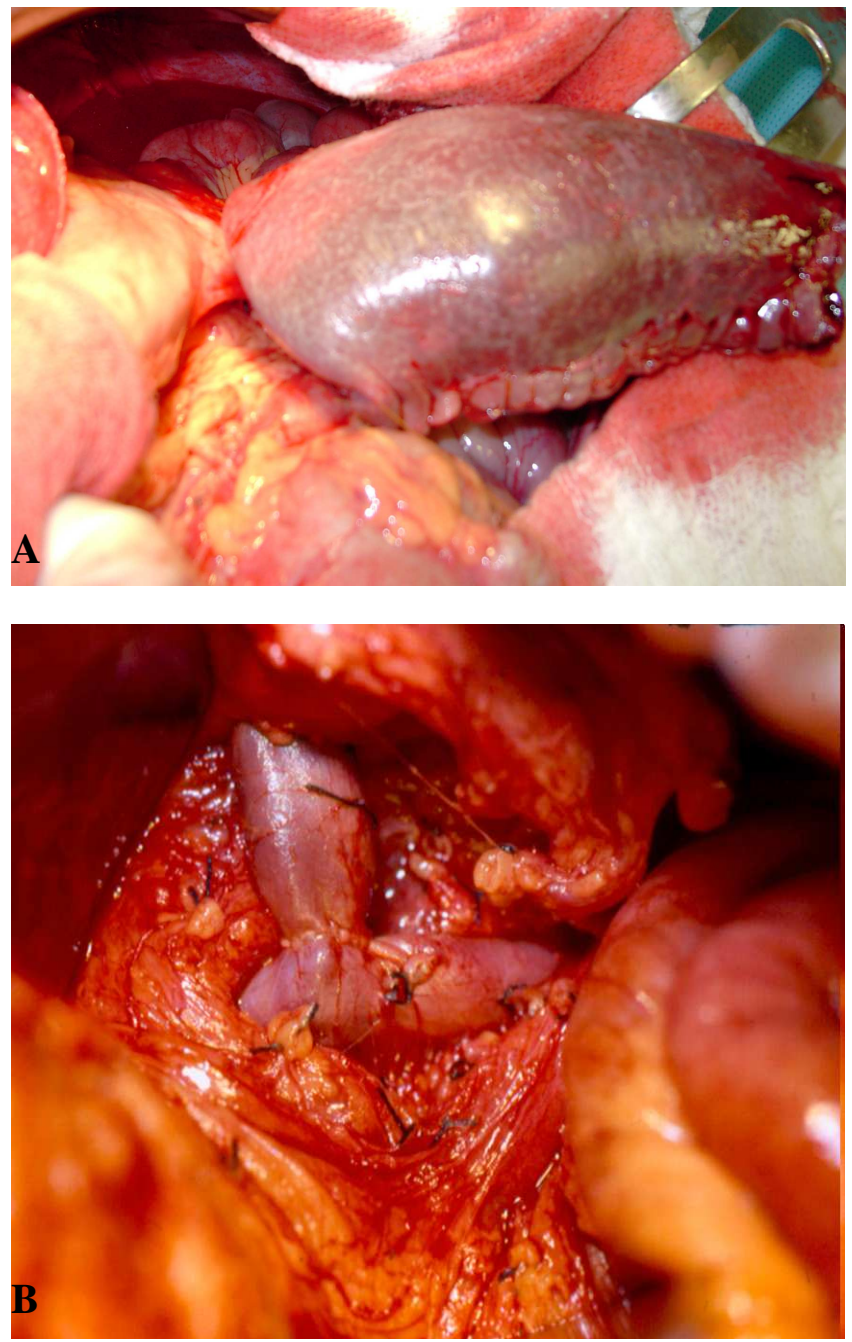

Figura 2 - Vista de tempos operatórios. A - Esplenectomia subtotal. Observar o remanescente esplênico (pólo superior) suprido apenas pelos vasos esplenogástricos. $\boldsymbol{B}$-Derivação término-lateral entre a veia esplênica (extremidade proximal) e a veia renal esquerda.

\section{RESULTADO}

A paciente evoluiu no pós-operatório sem intercorrências e recebeu alta hospitalar no terceiro dia pósoperatório. Após um seguimento de 30 meses, a paciente encontra-se assintomática. Recente avaliação com ultrasonografia e Doppler, evidenciou a anastomose esplenorrenal pérvia, com fluxo hepatopedal.

O remanescente esplênico permaneceu bem vascularizado e sem alteração em suas dimensões. $\mathrm{O}$ hemograma e o coagulograma permanecem dentro dos limites normais. Não há presença de corpúsculos hemáticos anômalos, que são usualmente encontrados após esplenectomia total. O exame cintilográfico confirmou a função esplênica de captação do radiofármaco.

\section{DISCUSSÃO}

A hemorragia proveniente de varizes intestinais é uma complicação muito rara da hipertensão porta, de difícil resolução, de acordo com a literatura. O presente trabalho apresenta o sexto caso descrito em que varizes localizadas em anastomose coledocojejunal provocam hemorragia grave e descreve um tratamento original e eficaz.

O sangramento provocado por varizes em pacientes com hipertensão porta é mais comum no esôfago e no estômago. A formação de varizes no intestino delgado tem a mesma fisiopatologia, porém não se acompanha de complicações ${ }^{5,7,10,26-30}$. Embora não esteja claro o motivo pelo qual varizes sangram, postula-se que aderências intra-abdominais em torno de áreas operadas sejam vias hepatofugas, elevando ainda mais a pressão local ${ }^{5,10,26-32}$.

A acurácia dos testes diagnósticos para detectar varizes sangrantes do intestino delgado é baixa. A angiotomografia, por exemplo, apesar de sua sensibilidade, tem acurácia de apenas $72 \%{ }^{33-35}$. Outros exames, como a cintilografia com hemácias marcadas com tecnécio 99m, têm uma sensibilidade de apenas $48 \%$. Já a arteriografia, que é o procedimento diagnóstico mais utilizado para detectar sangramento de intestino delgado, é capaz de indicar o sangramento somente se o exame for feito no momento da hemorragia e ela for superior a $1 \mathrm{ml} / \mathrm{min}$ 7,10,26,27,29,32,37,38,39 . Mesmo nessas condições, o diagnóstico pode ser feito com segurança em menos de $75 \%$ dos $\operatorname{casos}^{37}$.

Métodos endoscópicos, como esofagogastroduodenoscopia e a colonoscopia não detectam varizes de intestino delgado, no entanto, são exames importantes, para excluir a hemorragia proveniente dos locais por eles investigados. Exame endoscópico do intestino delgado, por endoscopia (push endoscopy - endoscopia por cápsula) ou enteroscopia intra-operatória é capaz de determinar a fonte de sangramento em $30 \%$ a $70 \%$ dos $\operatorname{casos}^{40-45}$. A enteroscopia intra-operatória tem a maior acurácia entre os métodos anteriores, no entanto, ela é indicada apenas quando métodos menos invasivos não determinam a fonte do sangramento ${ }^{46}$.

As opções de tratamento cirúrgico para varizes sangrantes do intestino delgado, descritas na literatura são ressecções intestinais, que podem ser associadas a derivações venosas ou sutura das varizes ${ }^{4,5,7,10,26-30}$. Contudo, há controvérsias sobre a melhor opção de tratamento cirúrgico. As derivações portassistêmicas têm, como vantagem, a redução pressórica no território das varizes, prevenindo assim novos sangramentos $5,7,10,30,47-49$.

Em contrapartida, as ressecções de segmentos intestinais e suturas das varizes acompanham-se de elevada taxa de recidiva ${ }^{5,7}$. No caso apresentado a sutura das varizes foi ineficaz, porém a derivação esplenorrenal proximal resolveu a hemorragia sem recorrências.

Ao associar-se a esplenectomia subtotal à descompressão do sistema porta, há o benefício de manter as importantes funções do baço. Essa operação já fora utilizada previamente, com bons resultados, para tratar sangramento de varizes esofágicas e gástricas, decorrentes de hipertensão porta esquistossmática ${ }^{12,13,50}$. Os resultados do caso aqui descrito confirmam achados prévios e reforçam a possibilidade de preservar parte do baço, mesmo após ligadura e secção dos vasos esplênicos principais.

Concluindo, pode-se considerar a esplenectomia subtotal, combinada com derivação esplenorrenal proximal, um tratamento adequado para controlar a hemorragia proveniente de varizes intestinais provocadas por hipertensão porta. 


\begin{abstract}
Background: One of the greatest challenges of portal hypertension treatment is the approach of severe variceal intestinal bleeding. The purpose of this article is to present subtotal splenectomy and splenorenal shunt as a new treatment of severe bleeding from a Roux en Y jejunal loop varices secondary to portal hypertension. Technique: A 64-year-old white woman presented severe episodes of bleeding from varices inside a Roux en Y jejunal loop as a complication of cirrhosis due to bile duct injury. The treatment included subtotal splenectomy with preservation of the upper splenic pole supplied only by the splenogastric vessels combined with an end-to-side central splenorenal shunt. Results: This procedure was safely performed with minor bleeding and no major technical difficulties or complications. A 30-month-post-operative-follow-up has been uneventful. No rebleeding occurred during this period and she returned to her normal life. Conclusion: Subtotal splenectomy combined with central splenorenal shunt seems to be a good option for the treatment of severe intestinal bleedings due to portal hypertension.
\end{abstract}

Key words: Splenectomy; Splenorenal shunt, surgical; Gastrointestinal bleeding; Esophageal and gastric varices; Portal hypertension.

\section{REFERÊNCIAS}

1. Katz LB. The role of surgery in occult gastrointestinal bleeding. Semin Gastrointest Dis. 1999;10(2):78-81.

2. Laine L. Upper gastrointestinal tract hemorrhage. West J Med. 1991;155(3):274-9.

3. Prediction of the first variceal hemorrhage in patients with cirrhosis of the liver and esophageal varices. A prospective multicenter study. The North Italian Endoscopic Club for the Study and Treatment of Esophageal Varices. N Engl J Med. 1988;319(15):983-9.

4. Salam AA, Goldman M, Smith D Jr, Hill HL. Gastric, intestinal and gallbladder varice: hemodynamic and therapeutics considerations. South Med J. 1979;72(4):402-8.

5. Wilson SE, Stone RT, Christie IP, Passaro E Jr. Massive lower gastrointestinal bleeding from intestinal varices. Arch Surg. 1979;114(10):1158-61.

6. Longacre AV, Gross CP, Gallitelli M, Henderson KJ, White RI Jr., Proctor DD. Diagnosis and management of gastrointestinal bleeding in patients with hereditary hemorrhagic telangiectasia. Am J Gastroenterol. 2003;98(1):59-65.

7. Moncure AC, Waltman AC, Vandersalm TJ, Linton RR, Levine FH, Abbott WM. Gastrointestinal hemorrhage from adhesionrelated mesenteric varices. Ann Surg. 1976;183(1):24-9.

8. Ricci RL, Lee KR, Greenberger NJ. Chronic gastrointestinal bleeding from ileal varices after total proctocolectomy for ulcerative colitis: correction by mesocaval shunt. Gastroenterology. 1980;78(5 Pt 1):1053-8.

9. Tang SJ, Zanati S, Dubcenco E, Cirocco M, Christodoulou D, Kandel G, et al. Diagnosis of small-bowel varices by capsule endoscopy. Gastrointest Endosc. 2004;60(1):129-35.

10. Yuki N, Kubo M, Noro Y, Kasahara A, Hayashi N, Fusamoto H, Ito T, Kamada T. Jejunal varices as a cause of massive gastrointestinal bleeding. Am J Gastroenterol. 1992;87(4):5147.

11. Petroainu A, Oliveira AE, Alberti LR. Hypersplenism in schistosomatic portal hypertension. Arch Med Res. 2005;36(5):496-501.

12. Petroianu A. Esplenectomia subtotal e anastomose esplenorrenal proximal para o tratamento da hipertensão portal. Rev Bras Cir. 1983;73(3):101-4.

13. Petroianu A. Treatment of portal hypertension by subtotal splenectomy and central splenorenal shunt. Postgrad Med J. 1988;64(747):38-41.

14. Petroianu A. Subtotal splenectomy and portal variceal disconnection in the treatment of portal hypertension. Can J Surg. 1993;36(3):251-4.
15. Petroianu A. Treatment of cystadenoma of the pancreatic tail by distal pancreatectomy and subtotal splenectomy. Dig Surg. 1995;12(4):259-61.

16. Petroianu A. Subtotal splenectomy in Gaucher's disease. Eur J Surg. 1996;162(6):511-3.

17. Petroianu A. Subtotal splenectomy for treatment of patients with myelofibrosis and myeloid metaplasia. Int Surg. 1996;81(2):177-9.

18. Petroianu A. Subtotal splenectomy for treatment of retarded growth and sexual development. Minerva Chir. 2003;58(3):4134.

19. Petroianu A. Subtotal splenectomy for the treatment of chronic lymphocytic leukemia. Ann Hematol. 2003;82(11):708-9. Epub 2003 Aug 2.

20. Petroianu A, Berindoague Neto R. Laparoscopic subtotal splenectomy. Minerva Chir. 2004;59(5):501-5.

21. Petroianu A, Silva RG, Simal CJ, Carvalho DG, Silva RA. Late postoperative follow-up of patients submitted to subtotal splenectomy. Am Surg. 1997;63(8):735-40.

22. Petroianu A, Petroianu LPG. A twelve-year-follow-up of subtotal splenectomy and central splenorenal shunt for treatment of schistosomatic portal hypertension. Folha Méd. 1998;116(3):107-9.

23. Petroianu A, Antunes LJ. Immune profiles in hepatosplenic schistosomiasis mansoni after surgical treatments. J Int Med Res. 1998;26(1):43-9.

24. Petroianu A, Resende V, Silva RG. Late follow-up of patients submitted to subtotal splenectomy. Int Surg. 2006;4(3):172-8. Epub Feb 20.

25. Resende V, Petroianu A. Functions of the splenic remnant after subtotal splenectomy for treatment of severe splenic injuries. Am J Surg. 2003;185(4):311-5.

26. Attias E, Smadja C, Vons C, Traynor O, Franco D. Bleeding from intestinal varices after a Warren shunt. J Clin Gastroenterol. 1987;9(5):585-7.

27. Joo YE, Kim HS, Choi SK, Rew JS, Kim HR, Kim SJ. Massive gastrointestinal bleeding from jejunal varices. J Gastroenterol. 2000; 35(10):775-8.

28. Migou S, Hashizume M, Tsugawa K, Kishihara F, Kwanaka H, Ohata M, et al. Jejunal variceal bleeding after esophageal transection in a patient with idiopathic portal hypertension. Hepatogastroenterology. 1998;45(20):5037.

29. Paquet KJ, Lazar A, Bickhart J. Massive and recurrent gastrointestinal hemorrhage due to jejunal varices in an afferent loop--diagnosis and management. Hepatogastroenterology. 1994;41(3):276-7. 
30. Smith AM, Walsh RM, Henderson JM. Novel bile duct repair for bleeding biliary anastomotic varices. J Gastrointest Surg. 2005;9(6):832-6.

31. Getzlaff S, Benz CA, Schilling D, Riemann JF. Enteroscopic cyanoacrylate sclerotherapy of jejunal and gallbladder varices in a patient with portal hypertension. Endoscopy. 2001;33(5):4624.

32. Sato T, Yasui O, Kurokawa T, Hashimoto M, Asanuma Y, Koyama K. Jejunal varix with extrahepatic portal obstruction treated by embolization using interventional radiology: report of a case. Surg Today. 2003;33(2):131-4.

33. Ettore GC, Francioso G, Garriba AP, Fracella MR, Greco A, Farchi G. Helical CT angiography in gastrointestinal bleeding of obscure origin. AJR Am J Roentgenol. 1997;168(3):727-31.

34. Rabe FE, Becker GJ, Besozzi MJ, Miller RE. Efficacy study of the small-bowel examination. Radiology. 1981;140(1):47-50.

35. Rex DK, Lappas JC, Maglinte DD, Malczewski MC, Kopecky KA, Cockerill EM. Enteroclysis in the evaluation of suspected small intestinal bleeding. Gastroenterology. 1989;97(1):58-60.

36. Olds GD, Cooper GS, Chak A, Sivak MV Jr, Chitale AA, Wong RC. The yield of bleeding scans in acute lower gastrointestinal hemorrhage. J Clin Gastroenterol. 2005;39(4):273-7.

37. Bloomfeld RS, Smith TP, Schneider AM, Rockey DC. Provocative angiography in patients with gastrointestinal hemorrhage of obscure origin. Am J Gastroenterol. 2000;95(10):2807-12.

38. Rollins ES, Picus D, Hicks ME, Darcy MD, Bower BL, Kleinhoffer MA. Angiography is useful in detecting the source of chronic gastrointestinal bleeding of obscure origin. AJR Am J Roentgenol. 1991;156(2):385-8.

39. Petroianu A. Transcatheter arterial embolization for hemorrhage caused by injury of the hepatic artery. J Gastroenterol Hepatol. 2005;20(7):973-4.

40. Ge ZZ, Hu YB, Xiao SD. Capsule endoscopy and push enteroscopy in the diagnosis of obscure gastrointestinal bleeding. Chin Med J. 2004;117(7):1045-9.

41. Gostout CJ. Improving the withdrawal phase of Sonde enteroscopy with the "push-away" method. Gastrointest Endosc. 1993;39(1):69-72.
42. Hayat M, Axon AT, O'Mahony S. Diagnostic yield and effect on clinical outcomes of push enteroscopy in suspected small bowel bleeding. Endoscopy. 2000;32(5):369-72.

43. Leighton JA, Triester SL, Sharma VK. Capsule endoscopy: a meta-analysis for use with obscure gastrointestinal bleeding and Crohn's disease. Gastrointest Endosc Clin N Am. 2006;16(2):229-50.

44. Lopez MJ, Cooley JS, Petros JG, Sullivan JG, Cave DR. Complete intraoperative small-bowel endoscopy in the evaluation of occult gastrointestinal bleeding using the sonde enteroscope. Arch Surg. 1996;131(3):272-7.

45. Morris AJ, Wasson L, MacKenzie JF. Small bowel enteroscopy in undiagnosed gastrointestinal blood loss. Gut. 1992;33(7):8879.

46. Petroianu A, Carvalho DG. Peroperative endoscopy in PeutzJeghers syndrome. Lancet. 1989;2(8676):1403.

47. Henderson JM. Portal hypertension and shunt surgery. Adv Surg. 1993;26:233-57.

48. Henderson JM. The role of portosystemic shunts for variceal bleeding in the liver transplantation era. Arch Surg. 1994;129(8):886.

49. Salmon PA. Small-bowel varices. Can J Surg. 1984;27(4):322.

50. Resende V, Petroianu A. Subtotal splenectomy for treatment of severe splenic injuries. J Trauma. 1998;44(5):933-5.

Como citar este artigo:

Petraoianu A, Rezende Neto JB. Tratamento de hemorragia intestinal grave decorrente de hipertensão porta, por meio de esplenectomia subtotal e anastomose esplenorrenal proximal - nota técnica. Rev Col Bras Cir. [periódico na Internet] 2008; 35(4). Disponível em URL: http://www.scielo.br/rcbc

Endereço para correspondência:

Prof. Andy Petroianu

Avenida Afonso Pena, 1626 - apto. 1901

30130-005 - Belo Horizonte - MG

Telefone / Fax: 31-3274-7744 e 8884-9192 (móvel)

E-mail: petroian@medicina.ufmg.br 